\title{
SPECTRAL PROPERTIES OF SOLAR CONVECTION AND DIFFUSION
}

\author{
A. A. Ruzmaikin, A. C. Cadavid, G. A. Chapman, J. K. Lawrence, and S. R. Walton \\ Department of Physics and Astronomy, California State University, Northridge, CA 91330-8268 \\ Received 1995 June 7 ; accepted 1996 June 10
}

\begin{abstract}
We present the results of a study of the scaling properties of solar photospheric motions. We use time series of Doppler images obtained in good seeing conditions with the San Fernando Observatory $28 \mathrm{~cm}$ vacuum telescope and vacuum spectroheliograph in video spectra-spectroheliograph mode. Sixty line-ofsight Doppler images of an area of the quiet Sun near disk center are investigated. They were taken at $60 \mathrm{~s}$ intervals over a $1 \mathrm{hr}$ time span at $\sim 2^{\prime \prime}$ resolution.

After filtering to remove 5 minute acoustic oscillations, the time-spatial spectrum of the velocity is calculated. To study the turbulence of photospheric flows in the mesogranulation scale range, we estimate two scaling parameters in the spectrum: the exponent of the spatial part of the power spectrum and the exponent governing the scaling of time correlations of each spatial mode. These parameters characterize the type of diffusion involved and the fractal dimension of the diffusion front. Our results indicate that the turbulent diffusion produced by motions in this scale range is not normal diffusion but superdiffusion.
\end{abstract}

Subject headings: convection - diffusion - methods: data analysis - Sun: interior

\section{INTRODUCTION}

It is generally agreed that the motions observed on the solar surface have a convective nature. Intensive studies of solar velocity spectra have been conducted in the past in order to identify characteristic structures in the solar convection, such as granules, mesogranules, and supergranules (Leighton, Noyes, \& Simon 1962; November et al. 1981). Knowledge of the underlying continuous velocity spectrum also is important for the study of basic solar phenomena, such as turbulent diffusion of magnetic fields or chaotic excitation of solar oscillations. For these purposes, we need the spacetime background spectrum, i.e., the distribution of energy over both scales and time intervals. The basic problem is that the convection, at least at some scales, is turbulent because of a very high Reynolds number. The character of the turbulence in the solar photosphere has not yet been fully established. However, we can cite the result by Muller (1989), who obtained the spatial spectrum of the solar line-of-sight velocity field in the scale range between granulation and supergranulation and found it to be consistent with the spectrum in Kolmogorov turbulence.

The present paper suggests a simple new tool for the study the solar turbulence by the use of observed time series of Doppler images. We present estimates of two key parameters of spacetime spectra of the solar velocity field over the range in which the spectra have power-law form. The first parameter is the well-known spectral exponent $(\alpha \leq 3)$ of the spatial correlation function. This measures the range of the spatial correlation of the velocity field. The second, less familiar exponent $(z>0)$ of the time correlation function for spatial Fourier modes measures the degree to which structures on larger spatial scales remain correlated longer than do those on smaller scales. Our investigation is made especially interesting by the discovery by Avellaneda \& Majda (1992a) that different regions of a two-dimensional " $\alpha-z$ diagram" contain very different kinds of turbulent diffusion. For example, when $\alpha<1-z$ (called region I), the long-range spatial correlation is weak and the normal Fickian diffusion occurs. On the other hand, when $\alpha>3-2 z$ (called region III), long-range spatial corre- lations are very strong. Here the diffusion is independent of the temporal correlation but it is nonstationary and superdiffusive, with an effective diffusion coefficient growing linearly in time. Region II, with $1-z<\alpha<3-2 z$, is intermediate and characterized by superdiffusive but stationary transport. The special case of Kolmogorov turbulence with $\alpha=5 / 3$ and $z=2 / 3$ lies exactly on the boundary between regions II and III. Thus, when they are known, the parameters $\alpha$ and $z$ can be used to characterize the turbulent diffusion of passive contaminants on the Sun and allow definition of the appropriate diffusivity. This turbulent diffusivity can also be used in the study of the magnetic field diffusion in the kinematic approximation, i.e., when the back action of the magnetic field on the velocity field is neglected. In this paper, an area of quiet-Sun network near the solar disk center is examined. Some preliminary results of a similar analysis of magnetic images of active regions can be found in Ruzmaikin et al. (1995).

As we shall see below, our observations are consistent with the conclusion that the solar velocity field has a power law from $2 \mathrm{Mm}$ to scales of at least $30 \mathrm{Mm}$ and that it lies in the $\alpha-z$ diagram near the Kolmogorov point on the boundary between regions II and III. Because it includes two independent parameters rather than one, this refines and strengthens the result by Muller (1989). Region I, which represents the normal, Fickian diffusion, is quite strongly excluded. Confirmation of such a result would have important implications for the evolution of solar surface magnetic fields over the applicable range of scales. It might also clarify why previous estimates of the magnetic turbulent diffusivity are so different

The paper is organized as follows. In $\S 2$, we define the two spectral exponents of the solar velocity field, $\alpha$ and $z$, and estimate their values from the observed series of Doppler images of the line-of-sight velocity. In $\S 3$, we use these exponents to examine the nature of solar turbulent diffusion in the framework of the Avellaneda \& Majda model. Section 4 presents another approach to the diffusion problem: the observational study of the geometrical properties of diffusion front. In particular, the fractal dimension of 
a diffusion front is defined by the same two spectral exponents, although here we are dealing directly with the horizontal fluid motions, the relationship of which to the line-of-sight motions is not precisely known. The relation of our results to the solar turbulent diffusion problem is discussed in $\S 5$.

\section{SPECTRUM OF THE TURBULENT VELOCITY FIELD}

Let a velocity field $v(x, t)$ be a random function of spatial coordinates and of time - in other words, a turbulent field. In the solar case, this approach appears to be valid for the scales smaller than supergranulation (Muller 1989; Chou et al. 1991). The supergranulation is also considered to be a random process over time intervals exceeding 15-30 hr supergranule cell lifetimes (Simon, Title, \& Weiss 1995). An essential property of turbulence is the existence of an inertial range of scales or wavenumbers $\left(k_{0}<k<k_{d}\right)$ located between the basic energy input scale $k_{0}^{-1}$ and the dissipation scale $k_{d}^{-1}$. In this range, the spatial spectrum of the velocity field is scale independent and therefore has a power-law form $k^{-\alpha}$ (Monin \& Yaglom 1975). Consider the Fourier modes of the line-of-sight velocity observed on the solar surface: $\quad \boldsymbol{v}(x, y, t)=\int \exp (\omega t-\boldsymbol{k} \cdot \boldsymbol{r}) d v(\boldsymbol{k}, t), \boldsymbol{k}=\left(k_{x}, \boldsymbol{k}_{y}\right)$. Then the correlation function corresponding to the powerlaw spectrum can be written in the form

$$
\left\langle d v(\boldsymbol{k}, t+\tau) d v^{*}(\boldsymbol{k}, t)\right\rangle=\left\langle v^{2}\right\rangle\left(\frac{k}{k_{0}}\right)^{-\alpha} f\left(\frac{\tau}{\tau_{c}}\right) \frac{d k_{x} d k_{y}}{k_{0} k},
$$

where $f$ is a function $[f(0)=1]$ that determines the temporal correlation properties of a Fourier mode with a given $\boldsymbol{k}$. It is assumed that, as in standard turbulence (Monin \& Yaglom 1975; Avellaneda \& Majda 1992a), $f$ is a decaying function and the characteristic time of its decay has a power-law form

$$
\tau_{c}(k)=\tau_{0}\left(\frac{k_{0}}{k}\right)^{z}
$$

Thus, the exponent $z>0$ defines the dependence of decorrelation times on the spatial scale. In particular, the longwavelength modes have longer decorrelation times.

Although scale-frequency studies of Doppler images have been conducted previously (Title et al. 1989; Chou et al. 1991), the parameter $z$ has not been explicitly determined. This definition of the time parameter, together with the attempt to characterize the turbulent transport (as opposite to simply evaluating the turbulent diffusivity), is the essential difference between these earlier studies and the present one. In the previous studies, the characteristic time was defined to be the correlation lifetime determined by measuring the $1 / e$ width of the temporal autocorrelation function averaged over space. We will use the exponent $z$, defining the correlation time for every Fourier mode (see eq. [2]). As we shall see in the application to the turbulent diffusion problem, this characteristic time carries important information about the velocity field.

In this work, we analyze 60 Doppler images of the line-ofsight velocity for a quiet-Sun region near disk center. A description of the instrumentation can be found in Chapman \& Walton (1989). The observations were made on 1994 September 14, at 1 minute intervals within $1 \mathrm{hr}$, starting at 20:25:00 UT. The images are composed of $470 \times 216$ pixels at 0.5 pixel $^{-1}$. An interpolation technique is used to cover a small number of bad records in three of the images (numbers 10, 45, and 52 in the sequence of images). A velocity image averaged over two cycles of the 5 minute oscillations is shown in Figure 1.

The images are aligned, and a fourth-order polynomial fit is used as a detrending technique. Because of the alignment process, and after eliminating bad pixels at the edges, the images used have a dimension of $450 \times 182$ pixels. We perform a two-plus-one-dimensional fast Fourier transform (FFT) on image sections of $128 \times 128 \times 60$ pixels, and we calculate the average energy spectrum from 20 such samples with random spatial locations. A Hanning spectral window is used in each case. To eliminate the $p$-mode oscillations, we use a subsonic filter, $\omega / k<7 \mathrm{~km} \mathrm{~s}^{-1}$ (Title et al. 1989; Chou et al. 1991). Then we calculate the energy spectrum as a function of $\omega$ and $k$. The energy spectrum of the velocity fields (Fig. 2) is defined from equation (2) as

$$
E(k) d^{2} k=\int k\left\langle d v(k, t+\tau) d v^{*}(k, t)\right\rangle d t,
$$

where $v(k, t)$ is the spatial FFT of the velocity, and the integral is taken over the whole 60 minute time interval. The exponent $\alpha$ is found by the fitting to $E(k) \propto k^{-\alpha}$ in the selfsimilar part of the spectrum. An estimate for the decorrelation time exponent $z$ is found by use of the relation

$$
E(k, \omega=0) \propto k\left\langle v(k, 0) v^{*}(k, 0)\right\rangle \propto k^{-\alpha-z} .
$$

Note that the spacetime spectrum at $\omega=0$ is independent of the form of unknown function $f\left(\tau / \tau_{c}\right)$. This spectrum is shown in Figure 3 in our approximation to $\omega=0$. The lowest frequency we can reach is, of course, 1/(60 minutes $)=1 /(3600 \mathrm{~s})$. To check to what extent the spectral exponents may be sensitive to this low cutoff of the frequency spectrum, we have made the same analysis for 180 Doppler images, also at 1 minute intervals. For this purpose, we have used a quiet region near the Sun's disk center, observed on 1994 September 23. We have found, within observational uncertainty, values of $\alpha$ and $z$ equivalent to those found for the 60 minute interval.

We estimate that the scaling range in Figures 2 and 3 extends up to the smallest frequency permitted by our sample sizes. (A feature appears superimposed on the power spectrum at $\sim 0.75 \mathrm{rad} \mathrm{Mm}^{-1}$, or at scale $\sim 8 \mathrm{Mm}$; however, we find it is not statistically significant.) The parameters found by the linear fits to 15 data points in the spectra of Figures 2 and 3 in the range $0.19 \mathrm{rad}$ $\mathrm{Mm}^{-1}<k<2.84 \mathrm{rad} \mathrm{Mm}^{-1}$ are

$$
\alpha=1.76\left\{\begin{array}{l}
-0.10 \\
+0.24
\end{array}\right\}, \quad z=0.38\left\{\begin{array}{l}
-0.24 \\
+0.45
\end{array}\right\} .
$$

These values exclude the possibility that we are in region I (normal diffusion) of the $\alpha-z$ phase diagram, but their uncertainties are large enough that they cannot tell us whether we are in region II or III (see Fig. 4).

The $\alpha$ and $z$ parameters have been calculated by linear fits to the plots shown in Figures 2 and 3. The uncertainties in the spectral data points are derived from averaging the spectra of $20128 \times 128 \times 60$ spacetime samples of our full data set. Individual linear fits are weighted according to these uncertainties (Bevington \& Robinson 1992). The asymmetric uncertainties given above for $\alpha$ and $z$ are derived from the "bootstrap" resampling scheme (Efron \& Tibshirani 1993), which gives a histogram (distribution function) for each parameter instead of just one value. Here we randomly choose a sample of 15 data points from the full set with replacement, so that some data points may be 


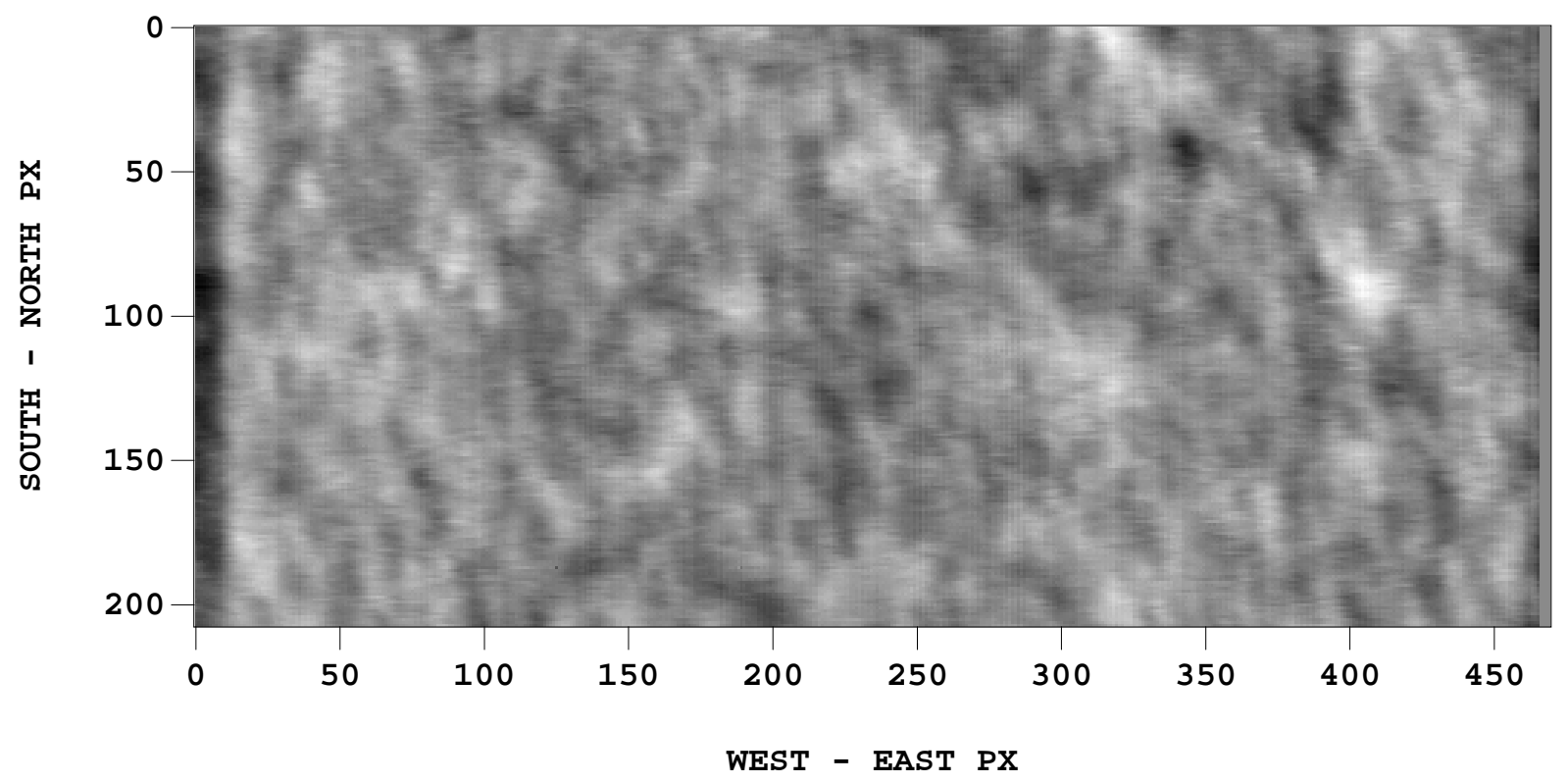

FIG. 1.-Doppler image from a series of 1994 September images obtained at San Fernando Observatory

repeated and others omitted. Then the weighted linear fit is computed. This procedure is repeated a large number of times ( 8192 in the present case). The value given for $\alpha$ or $z$ is the most frequently occurring value. The upper and lower uncertainties are chosen to exclude the upper and lower $16 \%$ of cases, and therefore they are analogous to the traditional $1 \sigma$ uncertainties. The asymmetry of the derived uncertainties indicates that a small number $(\sim 1)$ of possibly outlying data points are extremely influential in the fits. The bootstrap procedure gives a kind of hedge against that possibility. The values of $\alpha$ and $z$ given above are the most probable ones.

The range of wavenumbers in which the spectra appear self-similar corresponds to scales $(2 \pi / k)=2.22-33.2 \mathrm{Mm}$, i.e., about $2000-33,000 \mathrm{~km}$. This includes scales larger than

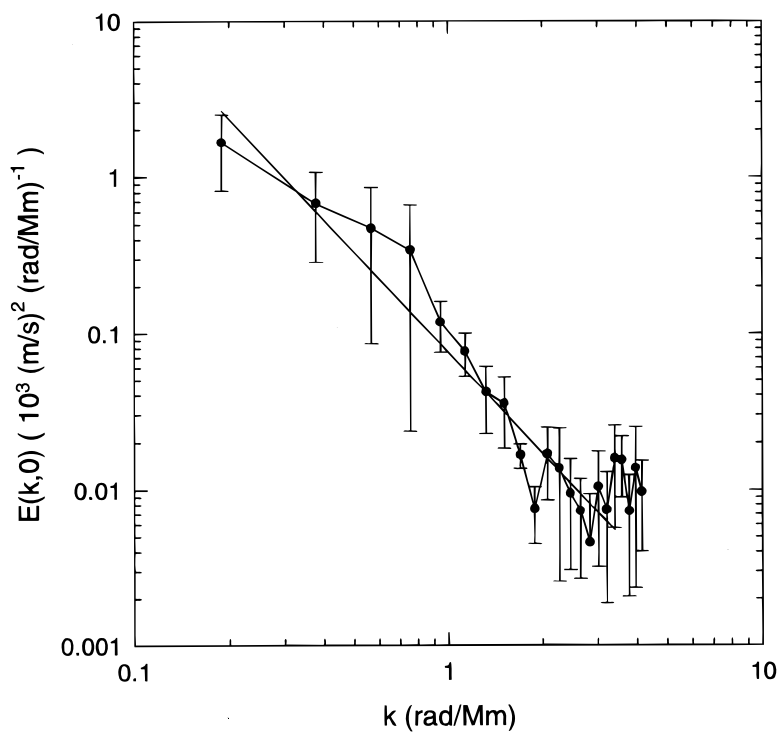

Fig. 2.-Spatial spectrum obtained with 60 images. Slope of this spectrum is $-\alpha$. Wavenumber is measured in $\mathrm{rad} \mathrm{Mm}^{-1}$. Power is measured in $\left.\left(\mathrm{m} \mathrm{s}^{-1}\right)^{2}(\mathrm{rad} \mathrm{Mm})^{-1}\right)^{-1}$.

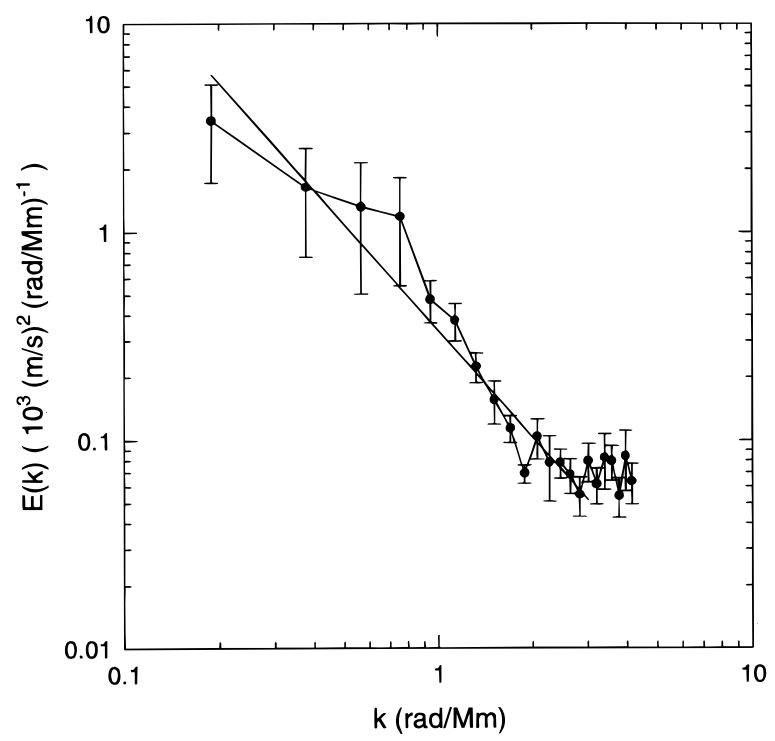

FIG. 3.-Spacetime spectrum at $\omega=0$. Slope of this spectrum gives $z$.

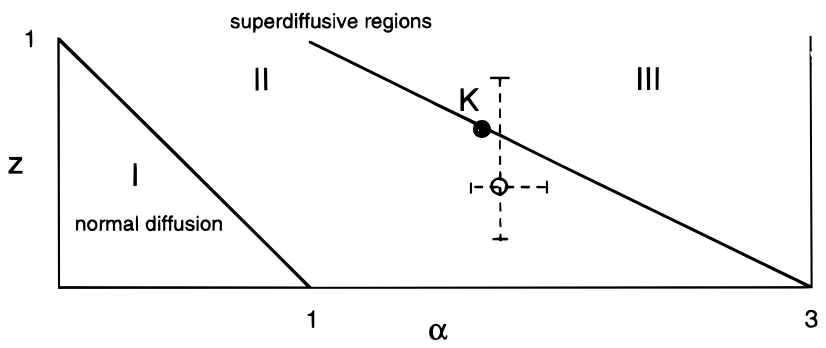

Fig. 4.-Diffusion diagram by Avellaneda \& Majda (1992a). Region I corresponds to normal Fickian diffusion. Regions II and III correspond to faster diffusion ("superdiffusion"). In region II, the temporal fluctuations are faster than the spatial fluctuations. In region III, the spatial fluctuations are the most important; this is a region of "frozen-in" turbulence. Kolmogorov regime is at the boundary between regions II and III. 
the granulation $(0.7-2 \mathrm{Mm})$ and extends beyond the mesogranulation $(2-11 \mathrm{Mm}$, with a peak at about $7 \mathrm{Mm})$. Note that the natural unit of measuring $k$ in our numerical calculations is the inverse size of a pixel. We convert this dimensionless $k$ into a wavenumber with the dimension of "radians per megameter," as is standard in solar physics. Our pixel size corresponds to 0.51 , and $1^{\prime \prime}$ subtends 0.72 $\mathrm{Mm}$ on the Sun. Thus, the conversion formula from the dimensionless $k$ to $k$ measured in $\mathrm{rad} \mathrm{Mm}^{-1}$ has the form:

$$
k \frac{\mathrm{rad}}{\mathrm{Mm}}=\frac{2 \pi}{N} \frac{1}{0.51 \mathrm{pixel}^{-1}} \frac{1}{0.72 \mathrm{Mm} \mathrm{arcsec}}-1
$$

where $N \times N$ is the number of pixels in the image. The energy spectra presented in Figures 2 and 3 are measured in units of $\left(\mathrm{m} \mathrm{s}^{-1}\right)^{2}\left(\mathrm{rad} \mathrm{Mm}^{-1}\right)^{-1}$.

\section{APPLICATION TO SOLAR TURBULENT DIFFUSION}

Turbulent diffusion is an important concept in the study of the transport of scalar quantities, such as temperature, and vector quantities, such as the large-scale solar magnetic field. It is a necessary element of the solar dynamo, which is the driving mechanism of solar activity (Parker 1979). Turbulent diffusion results from the presence of a wide spectrum of scales of motion. If the advected quantity is passive-i.e., does not affect the turbulence- then the character of the turbulent diffusion is defined by this motion. The advected quantity is assumed to have a characteristic scale exceeding the basic scale of turbulence.

It has become customary in solar studies of turbulent diffusion to use the dependence of the mean square distance on time in the form $\left\langle r^{2}\right\rangle \propto D t$, where $D$ is the turbulent diffusivity assumed to be constant. Although this is true for the normal, Fickian diffusion, there is no mathematical proof that shows that this dependence is valid in the turbulent case. Modern studies show that one must be careful when using the traditional mean square dependence of distance on time in the turbulent case. Here we discuss the model by Avellaneda \& Majda (1992a), in which the transport of a passive scalar $T$ in a given incompressible turbulent velocity field is considered:

$$
\frac{\partial T(x, t)}{\partial t}+v(x, t) \nabla T=0 .
$$

This equation contains the random functions $v(x, t)$. To reduce it to the regular diffusion equation, we must average over the turbulent motion. In this reduction, the action of the random velocity field is parameterized by an effective diffusivity. By means of a renormalization technique, Avellaneda \& Majda showed that the mean scalar $\langle T\rangle$ will evolve according to an effective diffusion equation if the coordinates and time are rescaled as $x \rightarrow x / \delta, t \rightarrow t / \delta^{1 / h}$, where the scaling factor $\delta$ is inversely proportional to the largest scale of the velocity spectrum. The parameter $h$, which defines the dependence of the mean square distance on time $\left\langle r^{2}\right\rangle^{1 / 2} \propto t^{h}$, can be derived from the spectral exponents $\alpha$ and $z$. (In Avellaneda \& Majda 1992a, the exponent $\epsilon=1+\alpha$ is used instead of $\alpha$.) In region II, the diffusion law has the exponent $h=1 /(3-\alpha-z)$. In region III, the diffusion law has the exponent $h=2 /(3-\alpha)$.

Thus, these two spectral exponents define the character of turbulent diffusion. Two basic regimes are identified depending on values of $\alpha$ and $z: h=\frac{1}{2}$ (normal diffusion), and $h>\frac{1}{2}$ (superdiffusion) (see Fig. 4).
Figure 4 shows the diffusion diagram by Avellaneda \& Majda (1992a), on which the Kolomogorov point (K) and the point found from our Doppler images are indicated. The observational point is close to or on the boundary between regions II and III. The turbulent diffusion in both regions II and III is "superdiffusive." The difference is that region II corresponds to the statistical regime in which timedecorrelation effects are dominant, whereas in region III time-decorrelation effects are negligible (the parameter $z$ does not come into play). In other words, region III is the regime of "frozen-in" turbulence in which temporal and spatial spectra are equivalent (the "Taylor hypothesis").

The concept of the superdiffusive turbulent diffusion is not new in hydrodynamics. Already in 1926, L. Richardson showed that in a turbulent fluid the mean square distance between two fluid particles grows as $t^{312}$ (Monin \& Yaglom 1975). However, Richardson had interpreted this diffusion as the ordinary diffusion, $r^{2}=D(r) t$, with a scale-dependent turbulent diffusivity $D \propto r^{4 / 3}$. We can interpret the Avellaneda \& Majda results in the same way by writing the turbulent diffusivity as $D(r)=D_{0}\left(r / r_{0}\right)^{2-1 / h}$. The Richardson case corresponds to $h=3 / 2$. The coefficient $D_{0}$, the value of the turbulent diffusivity at the basic scale $r_{0}$, can be calculated using of the values of the correlation time $\tau_{0}$ and the characteristic velocity $\left\langle v_{0}^{2}\right\rangle^{1 / 2}$ at this scale (see $\S 5$ ). A fundamental reason for the "superdiffusivity" is the non-Gaussian character of the random walk induced by turbulence - in particular, the significant probability of occasional very long steps (Schlesinger, West, \& Klafter 1987).

\section{LEVEL-SET DIMENSION}

The two parameters $\alpha$ and $z$ enter into many aspects of the transport of quantities advected by the turbulent motions. One such aspect is the fractal dimension $d$ of turbulent diffusion fronts or material interfaces - in other words, of surfaces advected by the small-scale motions of the fluid (Avellaneda \& Majda 1992b). In our case, since we are looking at two-dimensional cuts across the field distributions, we are interested in advected line elements. In region I, a Fickian diffusion front in a plane has dimension $d_{\mathrm{I}}=2$. In region II and on the boundary between regions II and III, the fractal dimension of such a line element is given by $d_{\text {II }}=(5-\alpha-z) / 2$. In region III, the fractal dimension is given by $d_{\mathrm{III}}=(5-\alpha) / 2$. These are discontinuous by an amount $z / 2$ across the boundary between regions II and III. Note that at the Kolmogorov point on the boundary, we expect $d_{\mathrm{K}} \equiv 4 / 3$.

Based on our estimated values for $\alpha$ and $z$, the predicted diffusion front dimensions in the two cases are $d_{\mathrm{II}}=$ $1.43\left\{\begin{array}{l}-0.02 \\ +0.22\end{array}\right\}$ and $d_{\mathrm{III}}=1.62\left\{\begin{array}{l}-0.05 \\ +0.12\end{array}\right\}$.

By assuming that the values of $\alpha$ and $z$-determined directly from line-of-sight motions - apply also to the corresponding horizontal motions (see, however, $\S 5$ ), we can try to determine the value of $d$ directly and therefore choose between regions II and III. Although the magnetic field is not a simple scalar contaminant, it is frozen to the high magnetic Reynolds number solar plasma and in the photosphere should be structured by the plasma motions. We have estimated the dimension of the interface between areas with line-of-sight field above and below a critical threshold, defined as follows. A histogram of the number of pixels in an image that have a given field value (in, for example, $1 \mathrm{G}$ bins) shows a Gaussian shape with standard deviation $\sigma$ for 


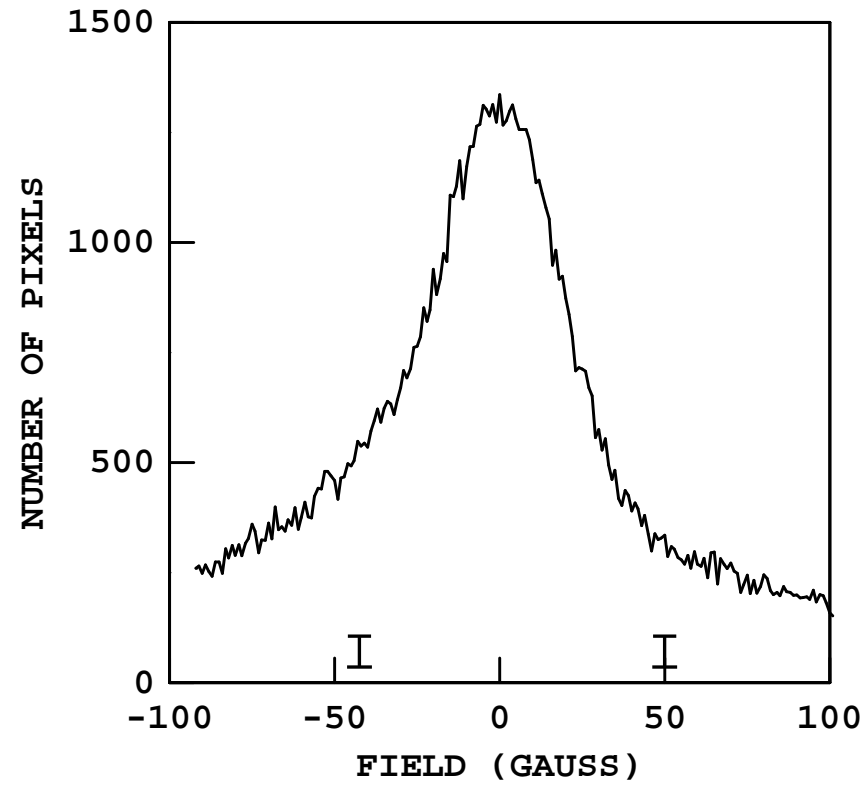

Fig. 5.-Frequency histogram of line-of-sight magnetic field values, in $1 \mathrm{G}$ bins for a San Fernando Observatory magnetic image made of NOAA active regions 6850 and 6853 on 1991 October 3 at UT 20:03:30. Spatial scale is 0.51 pixel $^{-1}$. Half-width of the Gaussian central peak is $\sigma=15 \mathrm{G}$. Markers at $B= \pm 3 \sigma= \pm 45 \mathrm{G}$ indicate the transition from the noise to the distribution wings representing the active region fields. weak fields. This Gaussian distribution may represent image noise, small-scale turbulent fields, or both, with field strength up to $\sim 3 \sigma$. The observed distribution for a San Fernando Observatory active region image with $\sigma=15 \mathrm{G}$ is shown in Figure 5. For magnetic fields $|B|>3 \sigma$, the Gaussian shape disappears and is replaced by broad wings representing the larger scale fields of both polarities. We define our interface to be comprised of those pixels with at least one pair of opposite nearest neighbors bracketing the value $B=3 \sigma$ (or, equivalently, $-3 \sigma$ ). The collection of such pixels comprise what is known as a "level set." Figure 6 shows both the positive and the negative level sets for the magnetic image of Figure 5.

In order to increase confidence that the level-set geometries are characteristic of the local nature of the fluid motions and not characteristic of the sources of the magnetic flux, we have examined a range of images. We have looked at images in which the field polarities are relatively segregated, as well as those in which they are more mixed. More importantly, we have studied images both of active regions and of network fields away from active regions. No systematic differences have been found. The main effect is that the network images contain less field and have a lower signal-to-noise ratio. Their level-set dimensions are therefore more widely scattered, though the average value is stable. This indicates that the level-set geometries are not

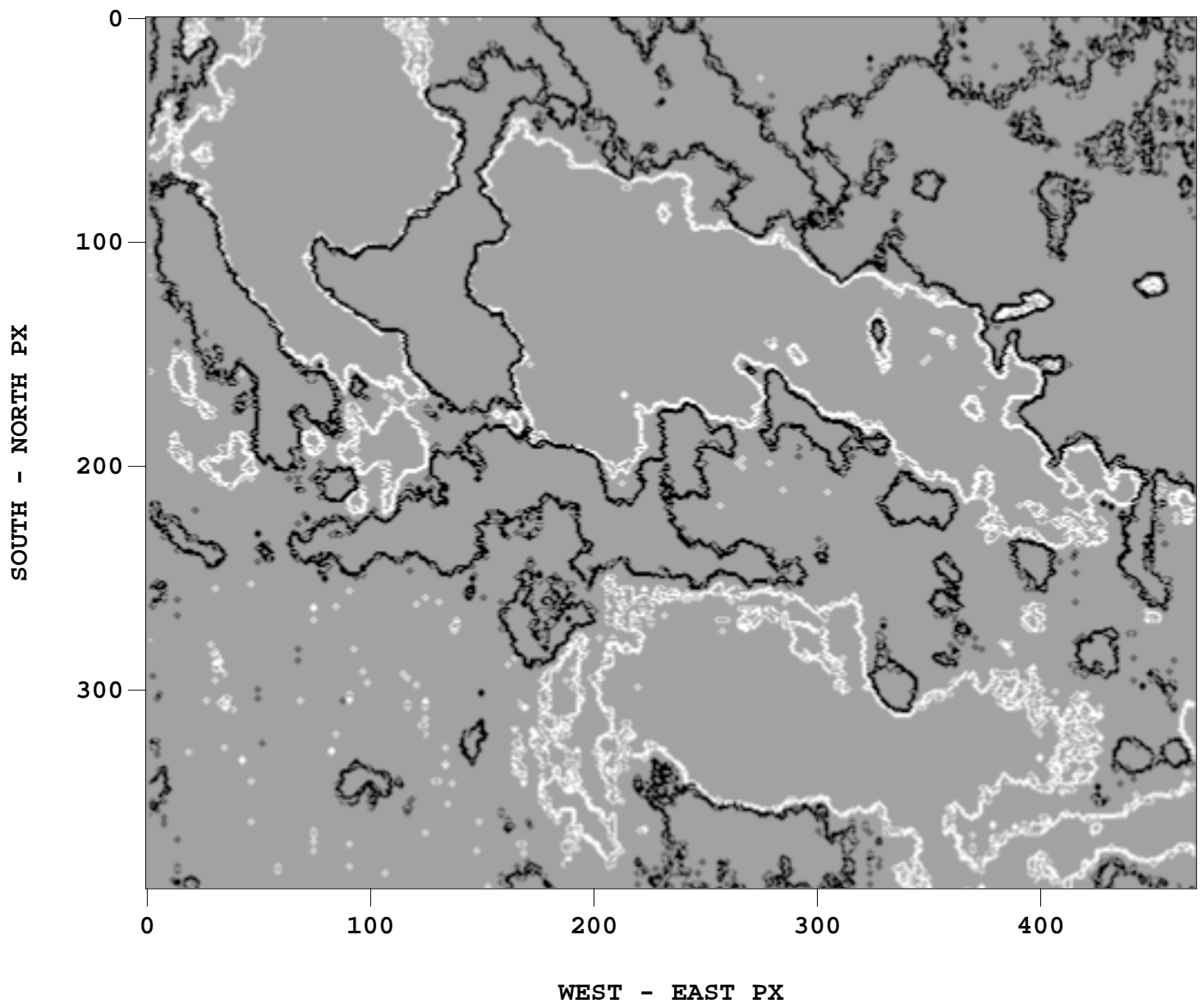

FIG. 6.-Map of the $B=+45 \mathrm{G}$ (white) and $B=-45 \mathrm{G}$ (black) level sets in a San Fernando Observatory magnetic image made of NOAA active regions 6850 and 6853 on 1991 October 3 at UT 20:03:30. Spatial scale is 0.51 pixel $^{-1}$; north is up, east is right. 


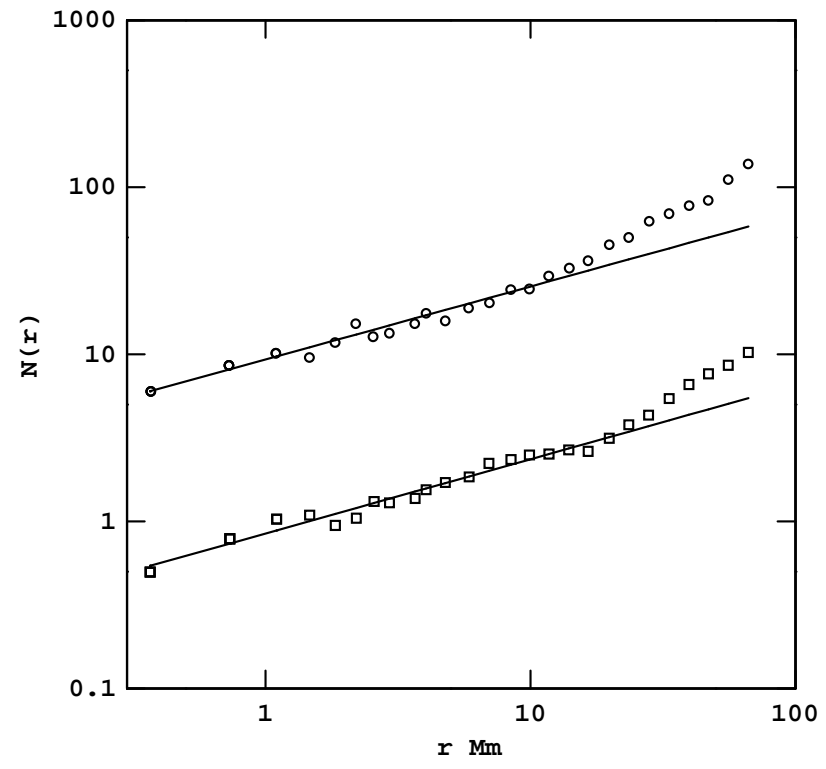

FIG. 7.- Log-log plot of the numbers $N(r)$ of pairs of points in level sets within a distance $r$ of one another. Slopes represent the fractal dimension of the set. Squares represent the positive polarity set in Fig. 6 and give $d_{+}=1.44$; circles, which have been shifted up by a factor of 10 for clarity, represent the negative polarity and give $d_{-}=1.46$.

significantly affected by the distribution of the field sources or by cancellation between opposite polarities. It is known that strong fields affect convective motions, and observations of random walks of magnetic flux elements (Schrijver \& Martin 1995) have given an effective diffusion coefficient in strong plage of 120 and $280 \mathrm{~km}^{2} \mathrm{~s}^{-1}$ in enhanced network. However, this difference need not characterize the basic geometry of the diffusion. Further study of the above data (Lawrence \& Schrijver 1993) showed that the random walks were characterized by a fractal dimension (different from the level-set dimension) that was the same in both cases.

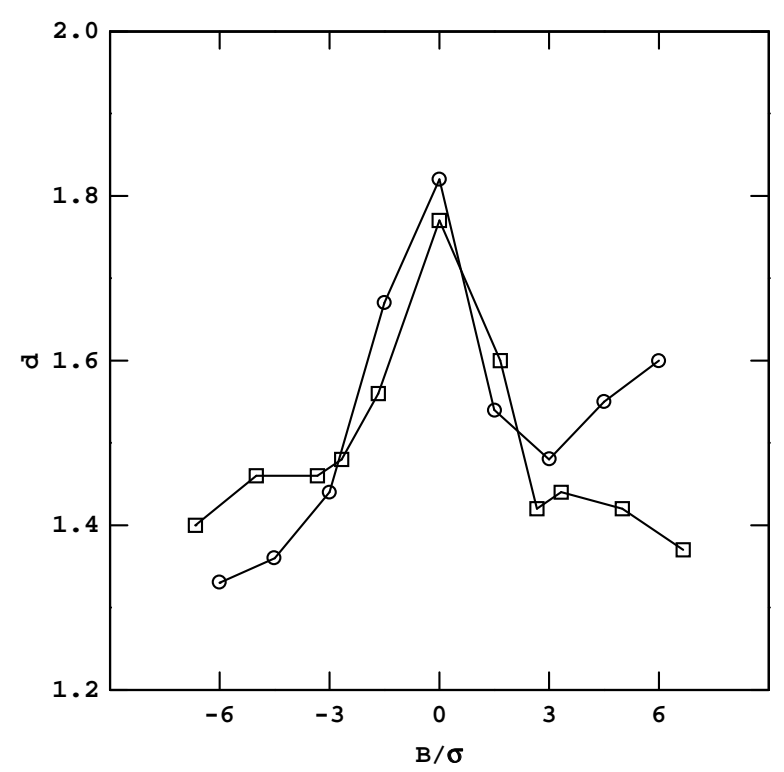

FIG. 8.-Fractal dimension of level sets vs. line-of-sight field level normalized to image noise amplitude. Squares represent the image described in Figs. 5 and 6. Circles represent a Lockheed-La Palma image of active region plage made on 1990 June 6 with a spatial scale of 0.14 pixel $^{-1}$.
The fractal dimensions of our level sets, including the two in Figure 6, were computed by a two-point correlation procedure in which the number of pairs of points of the set within a distance $r$ of one another scales as $N(r) \sim r^{d}$. Figure 7 shows log-log plots of $N(r)$ versus $r$ for the two level sets of Figure 6. For the positive level set we find $d_{+}=1.44$, and for the negative level set we find $d_{-}=1.46$. The horizontal scaling range over which these dimensions are seen to hold extends over more than a decade from the limit of resolution up to about $20 \mathrm{Mm}$, matching the top of the linear scaling range of the velocity power spectra from which $\alpha$ and $z$ were determined. In order to test the stability of the result, we have calculated the dimensions of several level sets for $-6 \sigma \lesssim B \lesssim+6 \sigma$ in some images. The result is shown in Figure 8 for the San Fernando Observatory image of Figures 5 and 6 (open squares) and also for a Lockheed-La Palma magnetogram (open circles). This indicates that for the range $3 \sigma<|B|<6 \sigma$, the fractal dimensions are relatively insensitive to the exact value of the level chosen. Note that the abscissae are normalized to the noise level $\sigma$, which corresponded to different field values in the two images: $15 \mathrm{G}$ for the San Fernando image, and $40 \mathrm{G}$ for the Lockheed-La Palma image.

Altogether, we have obtained estimates of 19 level sets from five San Fernando and two Lockheed-La Palma active region images, as well as from four San Fernando quiet-Sun images. The dimensions ranged from $d_{\min }=1.22$ to $d_{\max }=1.66$. The overall mean value was $d=1.43 \pm 0.02$. The values for network fields away from active regions showed a much higher scatter. They included both extreme values and gave a mean $d_{\text {net }}=1.42 \pm 0.08$. These results lead to the conclusion that we are dealing with plasma motions near the Kolmogorov point in region II of the phase diagram. Therefore, it is apparent that observations of relative motions of fluid markers - such as magnetic field elements - on the Sun can be valuable in characterizing the photospheric turbulence.

\section{DISCUSSION}

We have found estimates for two basic parameters of the background random velocity field in the wavenumber range $0.19 \mathrm{rad} \mathrm{Mm}^{-1}<k<2.84 \mathrm{rad} \mathrm{Mm}^{-1}$, which corresponds to the range of scales from $3 \times 10^{3}$ to $10.6 \times 10^{3} \mathrm{~km}$. These two parameters, $\alpha$ and $z$, can be used in a number of applications in which turbulence is present. As an example, we have evaluated the character of turbulent diffusion of a large-scale scalar or one-component quantity, such as the line-of-sight magnetic field. Because of the limited number of images, however, our spectral estimates have to be treated as preliminary.

The solar case is more complicated than the ideal model by Avellaneda \& Majda (1992a) in at least three aspects. First, the model is based on the assumption that the powerlaw behavior of the spectrum extends over a very wide range of scales. (Mathematically, they studied the asymptotics of very long inertial intervals at a large time intervals.) The inertial interval identified in the solar spectrum is finite; in fact, it is rather short (see Fig. 1). (Some reasons are the acoustic noise and low resolution at small scales, as well as the limited field of view.) Thus, any quantitative estimates we obtained - in particular, the values for the diffusion coefficient (see below) - must be considered as very approximate. However, the conclusion about the superdiffusive character of turbulent diffusion on the Sun based on the 
estimated values of the spectral parameters is probably correct because the normal diffusion requires a very small $\alpha$.

Second, the solar plasma is not an incompressible fluid. We have already removed one effect of compressibility, namely the acoustic noise. However, the effect of the density gradient on the velocity field was not taken into account in the model by Avellaneda \& Majda (1992a, 1992b). In incompressible flow, the spectral slopes of all components of the velocity vector are the same, so that characterizing the diffusion requires only one component of the velocity. The density gradient in the solar convective zone leads to a different scale dependence for the vertical and horizontal components of the velocity. If, in accordance with theoretical speculations, the ratio of vertical-to-horizontal velocities is defined by the mass conservation in a stratified convection, then $v_{v} / v_{h}$ varies from 0.6 for granulation to 0.03 for supergranulation (Chou et al. 1991). This means that the spectral slope for the horizontal component is expected to be even larger than the slope for the vertical component, found from our Dopplergrams. Thus, our basic conclusion about the "superdiffusive" character of the solar turbulent diffusion is expected to be valid when the effect of the density gradient is taken into account. Our study of horizontal level-set dimensions also supports this conclusion.

Third, solar turbulence is not hydrodynamic, it is magnetohydrodynamic. The large-scale magnetic field is a vector, and the magnetic force may well affect the turbulent motions. Thus, formally, we have no right to use passive scalar transport theory. However, passive scalar diffusion can be a good first approximation to the diffusion of the magnetic field (Parker 1979) or, at least, the line-of-sight component of the solar magnetic field. The back action of the magnetic field on the velocity requires additional studies.

The model by Avellaneda \& Majda (1992a, 1992b) allows also the calculation of the turbulent diffusivity $D_{0}$. For example, in region II, $D_{0}$ is given by $D_{0}=\left\langle v_{0}^{2}\right\rangle \tau_{0} / \pi(\alpha+z$ -1 ), where $v_{0}, \tau_{0}$ are the characteristic velocity and correlation time at the basic scale of the inertial interval, respectively. Thus, to estimate the value of the turbulent diffusivity we need to know the characteristic parameters at the basic scale. In fact, we need to know the basic scale and the value of velocity at this scale because $\left\langle v_{0}^{2}\right\rangle \tau_{0}=r_{0} v_{0}$. The basic scale has to be determined independently. Thus, Komm, Howard, \& Harvey (1995), analyzing the motions of magnetic elements on the solar surface, have found a characteristic scale of about $17 \mathrm{Mm}$, which is less than the supergranulation size. The velocity amplitude must include both the vertical and horizontal components, and from our Dopplergrams we know only the vertical component of the velocity. At the upper end of the inertial range, the horizontal velocity, which is poorly known, is dominant. For example, in the study of the turbulent diffusion produced by supergranulation, Simon et al. (1995) consider horizontal velocities in the range $0.2-1 \mathrm{~km} \mathrm{~s}^{-1}$. Using the end of our inertial interval $l_{0} \approx 10^{4} \mathrm{~km}$, which is close to the scale found by Komm et al. (1995), and $v_{0}=0.2 \mathrm{~km} \mathrm{~s}^{-1}$ at this scale, we obtain $D_{0} \approx 400 \mathrm{~km}^{2} \mathrm{~s}^{-1}$. This value, of course, depends on the estimate of the horizontal component of the velocity.

The known empirical estimates of the solar turbulent diffusivity are uncertain They depend on the methods and the parameters different authors use and vary between $10^{2}$ and $10^{3} \mathrm{~km}^{2} \mathrm{~s}^{-1}$ (Spruit, Norlund, \& Title 1994). For example, the value of the diffusivity estimated from studies of supergranulation is about $600 \mathrm{~km}^{2} \mathrm{~s}^{-1}$ (Wang \& Sheeley 1993; Simon et al. 1995). The value is found to be $120-250 \mathrm{~km}^{2}$ $\mathrm{s}^{-1}$ from the study of motions of magnetic elements (Schrijver \& Martin 1995; Komm et al. 1995). In the latter paper, $\tau_{0}$ was estimated as the lifetime of the magnetic elements. Taking into account the fact that magnetic elements live longer than the characteristic time of the random motions would obviously shorten the gap between the different estimates. It is not, however, a goal of the present paper to give a better estimate of the diffusivity. Instead, we want to attract attention to the fact that the solar turbulent diffusion is different from the normal Fickian diffusion. As a consequence, two quantities have to found from the observations: the exponent $h$ that defines the behavior of the mean square distance, and the turbulent diffusivity at the basic scale. At scales larger than the supergranulation, the supergranulation itself is random and acts diffusively with its own turbulent diffusivity. The type of this diffusion (normal or otherwise) must also be determined by future studies. All these factors have to be taken into account in the interpretation of the observations aimed at the study of turbulent diffusion.

The extension of the observational range is needed, especially to strengthen our knowledge of the temporal correlations of the line-of-sight and transverse photospheric velocity fields. A good way to expand the present study would be the use of space-based observations. The space measurements avoid the atmospheric distortions intrinsic to ground-based observations and therefore facilitate coregistration of images taken over time. In addition, accurate measurement of the temporal correlation exponent $z$ requires reliable observations of the decorrelation of velocity features over the longest possible time span. In the case of ground observations, this time span will be limited by the duration of intervals of good seeing - typically a few hours at best. Space-based observations eliminate this restriction. A unique opportunity is offered by the SOHO-MDI experiment (Scherrer 1995) that will provide a series of line-ofsight velocity images at $60 \mathrm{~s}$ intervals for up to $8 \mathrm{hr}$, complemented by measurements of the transverse velocity components.

We thank S. Molchanov and L. Peterbarg for discussions of mathematical problems related to the turbulent diffusion, and we thank the referee for helpful comments. This work has been supported in part by NSF grants ATM 93-09901, ATM 91-15111, and ATM 95-04374.
Avellaneda, M. \& Majda, A. J. 1992a, Phys. Rev. Lett. 68, 3028

Avellaneda, M., \& Majda, A. J. 1992a, Phys. Re

Bevington, P. R., \& Robinson, D. K. 1992, Data Reduction and Error Analysis for the Physical Sciences (2nd ed.; New York: McGraw-Hill)

Chapman, G. A., \& Walton, S. R. 1989, in High Spatial Resolution Solar Observations, ed. O. van der Luhe (Sunspot: NSO), 402

Chou, D.-Y., LaBonte, B. J., Braun, D. C., \& Duvall, T. L. 1991, ApJ, 372 314

\section{REFERENCES}

Efron, B., \& Tibshirani, R. J. 1993, An Introduction to the Bootstrap, Monographs on Statistics and Applied Probability No. 57 (New York: Chapman \& Hall)

Komm, R. W., Howard, R. F., \& Harvey, J. W. 1995, Sol. Phys., 158, 213 Lawrence, J. K. \& Schrijver, C. J. 1993, ApJ, 411, 402

Leighton, R. B., Noyes, R. W., \& Simon, G. W. 1962, ApJ, 135, 474

Monin, A. S., \& Yaglom, A. M. 1975, Statistical Fluid Mechanics: Mechanics of Turbulence Vol. 2 (Cambridge: MIT Press) 
Muller, R. 1989, in Solar and Stellar Granulation, ed. R. J. Rutten \& G. Severino (Dordrecht: Kluwer), 101

November, L. J., Toomre, J., Gebbie, K. B., \& Simon, G. W. 1981, ApJ, 245 L123

Parker, E. N. 1979, Cosmic Magnetic Fields (Oxford: Oxford Univ. Press)

Ruzmaikin, A. A., Cadavid, A. C., Chapman, G., Lawrence, J. K., \& Walton, S. 1995, in ASP Conf. Ser. 76, Gong '94: Helio-and AsteroSeismology from the Earth and Space, ed. R.K. Ulrich, E. J. Rhodes, Jr., \& W. Däppen (San Francisco: ASP), 292
Scherrer, P. 1995, in Fourth SOHO Workshop, Helioseismology, ed. T. Hoeksema (Dordrecht: Kluwer)

Schlesinger, M. F., West, B. J., \& Klafter, J. 1987, Phys. Rev. Lett, 58, 1100 Schrijver, C. J., \& Martin, S. 1990, Sol. Phys., 129, 95

Simon, G. W., Title A. M., \& Weiss, N. O. 1995, ApJ, 442, 886

Spruit, H. C., Norlund, \& Title, A. 1994, ARA\&A, 28, 263

Title, A. M., Tarbell, T. D., Topka, K. P., Ferguson, S. H., \& Shine, R. A. 1989, ApJ, 336, 475

Wang, H. C., \& Sheeley, N. R., Jr. 1993, ApJ, 414, 916 